\title{
Epigenetic Histone Deacetylation Inhibition Prevents the Development and Persistence of Temporal Lobe Epilepsy
}

\author{
Sandesh D. Reddy, Bryan L. Clossen, and Doodipala Samba Reddy \\ Department of Neuroscience and Experimental Therapeutics, Texas A\&M University Health Science Center, College of Medicine, \\ Bryan, Texas
}

Received August 31, 2017; accepted October 31, 2017

\section{ABSTRACT}

Epilepsy is a chronic brain disease characterized by repeated unprovoked seizures. Currently, no drug therapy exists for curing epilepsy or disease modification in people at risk. Despite several emerging mechanisms, there have been few studies of epigenetic signaling in epileptogenesis, the process whereby a normal brain becomes progressively epileptic because of precipitating factors. Here, we report a novel role of histone deacetylation as a critical epigenetic mechanism in epileptogenesis. Experiments were conducted using the histone deacetylase (HDAC) inhibitor sodium butyrate in the hippocampus kindling model of temporal lobe epilepsy (TLE), a classic model heavily used to approve drugs for treatment of epilepsy. Daily treatment with butyrate significantly inhibited HDAC activity and retarded the development of limbic epileptogenesis without affecting after-discharge signal. HDAC inhibition markedly impaired the persistence of seizure expression many weeks after epilepsy development. Moreover, subchronic HDAC inhibition for 2 weeks resulted in a striking retardation of epileptogenesis. HDAC inhibition, unexpectedly, also showed erasure of the epileptogenic state in epileptic animals. Finally, butyrate-treated animals exhibited a powerful reduction in mossy fiber sprouting, a morphologic index of epileptogenesis. Together these results underscore that HDAC inhibition prevents the development of TLE, indicating HDAC's critical signaling role in epileptogenesis. These findings, therefore, envisage a unique novel therapy for preventing or curing epilepsy by targeting the epigenetic HDAC pathway.

\section{Introduction}

Epilepsy, which affects about 3 million Americans and 65 million people worldwide, is a common brain disease characterized by repeated unprovoked seizures. Despite such wide incidence and the dozens of drugs available to combat seizures, there is currently no specific drug for preventing or curing epilepsy (Jacobs et al., 2009). About 150,000 new cases of epilepsy are diagnosed in the United States annually (Hesdorffer et al., 2013). The mechanisms underlying the development of acquired epilepsy are not very well understood. The term epileptogenesis is used to describe the complex plastic changes in the brain that, following a precipitating event, convert a normal brain into a brain debilitated by recurrent seizures (Pitkänen et al., 2015). Temporal lobe epilepsy (TLE) is caused by diverse precipitating factors such as brain injury, stroke, infections, or prolonged seizures. TLE progression can be described in three stages: 1) the initial epileptogenic event, 2) the latent period with little or no seizure activity, and 3) the chronic

The authors declare no competing financial interests

This work was partly supported by the Office of the Assistant Secretary of Defense for Health Affairs through the FY 2015 Epilepsy Research Program under Award W81XWH-16-1-0660 (to D.S.R.). The opinions, interpretations, conclusions, and recommendations are those of the author and are not necessarily endorsed by the U.S. Department of Defense.

https://doi.org/10.1124/jpet.117.244939. period characterized by recurrent seizures. The kindling model has provided a conceptual framework for epileptogenesis and developing new molecular targets to prevent epilepsy (Goddard et al.,1969; McNamara et al., 1992). Kindling is the classic model for epileptogenesis, in which repeated stimulation below the convulsion threshold leads to progressive intensification of behavioral and electrographic seizure activity (Goddard et al., 1969). It has become a widely used model for human TLE with complex partial seizures (Löscher, 2002; He et al., 2004; Gorter et al., 2016). There is an unmet need for drugs that truly prevent the development of epilepsy, known as antiepileptogenic agents, or alter its natural course to limit the appearance or severity of epileptic seizures, known as disease-modifying agents, in people at risk. Several antiepileptic drugs such as levetiracetam, topiramate, lamotrigine, and lacosamide have been tested for disease-modifying activity but have exhibited little efficacy (Albertson et al., 1984; Silver et al., 1991; Löscher et al., 1998; Brandt et al., 2007; Pitkänen et al., 2013). A variety of potential treatments have been proposed, targeting mechanisms such as the neurotrophic, antiinflammatory, neuroprotective, and epigenetic pathways (Pitkänen and Lukasiuk, 2011; Ravizza et al., 2011; Clossen and Reddy, 2017; Younus and Reddy, 2017a). However, there have been few studies on the potential role of histone deacetylase (HDAC) signaling in epileptogenesis.

ABBREVIATIONS: AD, after discharge; ADT, after-discharge threshold; GX, ganaxolone (3 $\beta$-methyl-3 $\alpha$-hydroxy- $5 \alpha$-pregnan-20-one); HDAC, histone deacetylase; TLE, temporal lobe epilepsy. 


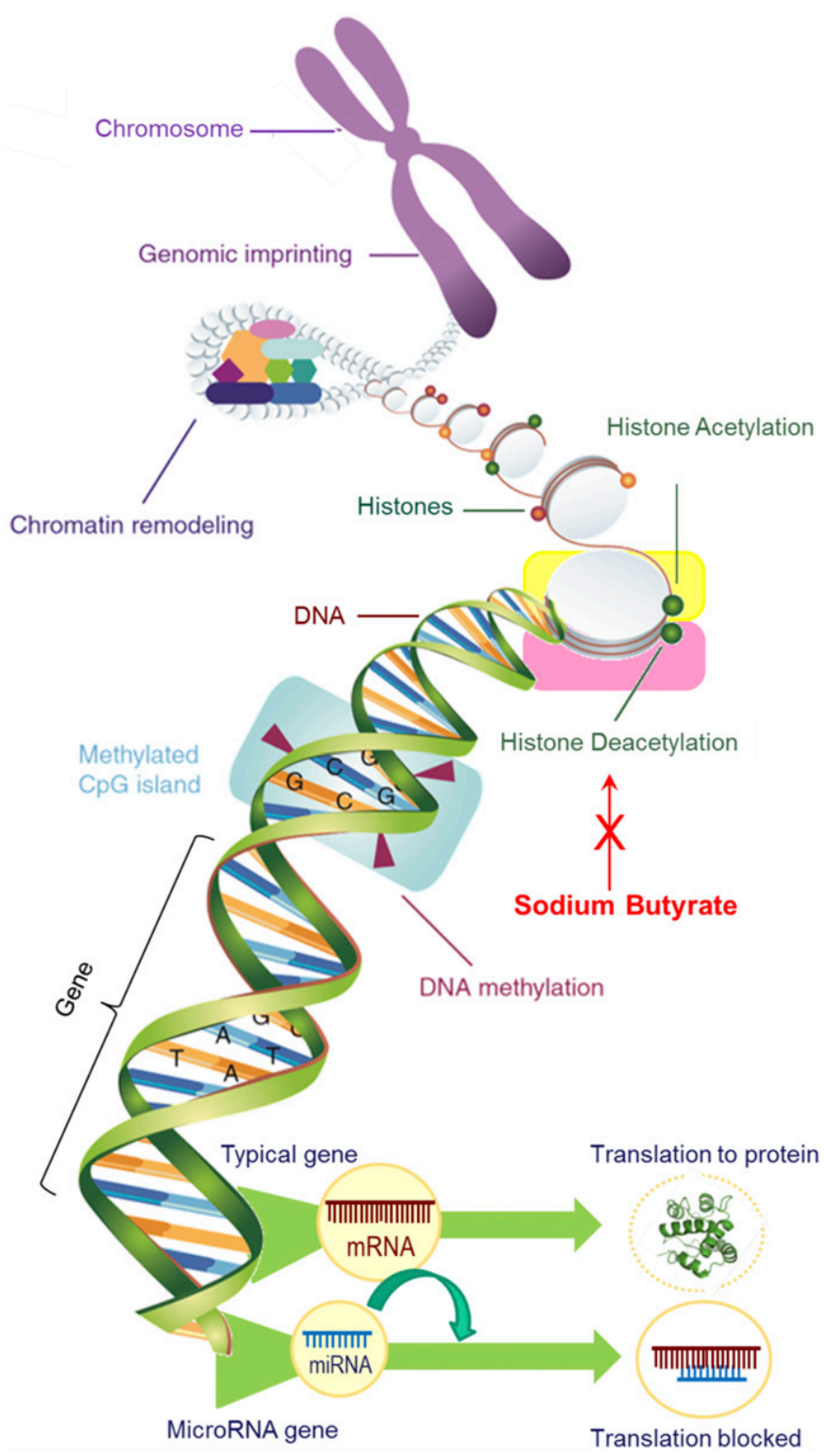

Fig. 1. Epigenetic pathway and HDAC sites of sodium butyrate's action. Sodium butyrate inhibits the HDAC 1,2 , and 7 enzymes, thus interfering with chromatin structure and reducing expression of genes involved in epileptogenesis by influencing several DNA transcription factors to regulate gene expression during transcription in the brain. Modified with permission from Qureshi and Mehler (2010b).

The potential epigenetic mechanisms, including DNA methylation, histone alterations, and microRNA-based transcriptional control, can alter the neuronal gene expression profile and thus affect epilepsy pathology (Fig. 1). Although epigenetic signaling alterations are evident in epileptogenesis (Qureshi and Mehler, 2010a; Younus and Reddy, 2017b), there has been little effort for drug discovery to target specific epigenetic mechanisms. This epigenetic approach, however, is broader than typical antiseizure treatments and represents a mechanistic strategy for preventing TLE. Histone acetylation is catalyzed by histone acetyltransferases, and histone deacetylation is carried out by HDACs. Generally, acetylation of histone lysine residues is associated with transcriptional activation (Verdone et al., 2005; Garcia-Bassets et al., 2007). Histone modifications can play a role in epilepsy (Younus and
Reddy, 2017b). Therefore, interruption of the HDAC pathway may provide a targeted strategy to prevent epilepsy progression. Sodium butyrate is a broad inhibitor of multiple HDACs from classes 1, 2, and 7 (Gurvich et al., 2004; Pajak et al., 2007). It acts similarly to, but has a wider range than, sodium valproate, which inhibits HDACs from classes 1 and 2 only. Sodium butyrate, a low molecular weight volatile fatty acid that was originally discovered for prevention of cancer, is a specific inhibitor of HDAC activity and can affect the expression of many mammalian genes (Davie, 2003) (Fig. 1). Thus, butyrate is commonly used as a specific epigenetic tool for interrupting HDAC signaling. Sodium butyrate crosses the blood-brain barrier and inhibits HDAC activity, inducing hyperacetylation of core histones that allow DNA binding of transcription factors to inhibit induction or repression of gene expression in the brain (Minamiyama et al., 2004; Deutsch et al., 2008; Kim et al., 2013; Takuma et al., 2014).

In the present study, we investigated the functional role of the HDAC pathway in experimental models of limbic epileptogenesis and TLE. Our results suggest that HDAC inhibition leads to a striking interruption of the development and persistence of epileptogenesis in a kindling model of TLE.

\section{Materials and Methods}

Animals. Young male wild-type C57BL/6 mice, 25-30 g each, were used in this study. All mice were housed individually in cages with access to food and water ad libitum. The mice were housed in an environmentally-controlled animal facility with a 12-hour light/dark cycle. The animals were cared for in strict compliance with the guidelines outlined in the National Institutes of Health Guide for the Care and Use of Laboratory Animals (https://www.ncbi.nlm.nih. gov/books/NBK54050/). All animal procedures were performed in a protocol approved by the university's Institutional Animal Care and Use Committee.

HDAC Activity Assay. To determine HDAC activity and its inhibition by sodium butyrate treatment, brain tissue samples were extracted from control and butyrate-treated $(600 \mathrm{mg} / \mathrm{kg}$, i.p.) mice. HDAC activity was measured from nuclear lysates using the Fluor-deLys HDAC activity assay kit (Biomol, Plymouth Meeting, PA), as reported previously (Nian et al., 2009; Rajendran et al., 2011). Briefly, incubation was performed at $37^{\circ} \mathrm{C}$ for 30 minutes with either cortex or hippocampal tissue lysates (15 $\mu \mathrm{g}$ protein/well), and the HDAC reaction was initiated by the addition of Boc-Lys(Ac)-AMC substrate. After a trypsin developer was added, the mixture was incubated for another 15 minutes at room temperature. In some experiments, the protein extracts from cortical or hippocampal tissues were treated with trichostatin A, a standard HDAC inhibitor, and then samples were assayed for HDAC activity. The addition of a trypsin-containing developer allows the product to be converted into a quantifiable fluorophore. Fluorescence was measured using a Synergy 2 plate reader (BioTek, Winooski, VT), with 360-nm excitation and 460-nm emission. The data were normalized to samples containing trichostatin A. HDAC enzymes convert the acetylated substrate, Boc-Lys(Ac)AMC, to a deacetylated product. HDAC inhibitors, such as sodium butyrate, can inhibit such a reaction.

Classic Hippocampus Kindling Model of Epileptogenesis. To study the antiepileptogenic activity of sodium butyrate, we used the hippocampus kindling model, which is the best model of human TLE, characterized by progressive complex partial seizures with secondary generalization (Goddard et al., 1969; Reddy et al., 2010). A mild focal, nonconvulsant electrical stimulus to the hippocampus on a daily basis leads to the development of a kindled state exhibiting electrographic and behavioral seizures. In mouse kindling, the focal electroencephalogram afterdischarge $(\mathrm{AD})$ models complex partial 
seizures, whereas behavioral motor seizure stages 4 and 5 model generalized seizures. Electrode implantation and stimulation procedures for mouse hippocampus kindling were performed as described previously (Reddy and Mohan, 2011; Reddy et al., 2012). Mice were anesthetized by an intraperitoneal injection of a mixture of ketamine $(100 \mathrm{mg} / \mathrm{kg})$ and xylazine $(10 \mathrm{mg} / \mathrm{kg})$. A twisted bipolar stainless-steel wire electrode (model MS303/1; Plastic Products, Roanoke, VA) was stereotaxically implanted in the right hippocampus $(2.9 \mathrm{~mm}$ posterior and $3.0 \mathrm{~mm}$ lateral to bregma and $3.0 \mathrm{~mm}$ below the dorsal surface of the skull) using the Franklin and Paxinos atlas (Franklin and Paxinos, 1997) and anchored with dental acrylic to four jeweler's screws placed in the skull. A period of 7-10 days was allowed for recovery. The stimulation paradigm consisted of 1-millisecond duration, bipolar, square current pulses delivered at $60 \mathrm{~Hz}$ for 1 second using a kindling stimulator (A-M Systems, Sequim, WA). The AD threshold (ADT) was determined by stimulating at 15-minute intervals beginning with an intensity of $25 \mu \mathrm{A}$ and increasing in steps of $25 \mu \mathrm{A}$ until an $\mathrm{AD}$ was obtained. Stimulation on subsequent days used a stimulation intensity of $125 \%$ threshold value. The $\mathrm{AD}$ was recorded from the hippocampus electrode with a Grass CP511 preamplifier (Astro-Med, West Warwick, RI) and stored in digital form using Axoscope 8.1 software (Axon Instruments, Foster City, CA). The AD duration was the total duration of hippocampal electrographic spike activity (amplitude $>2 \times$ baseline) occurring in a rhythmic pattern at a frequency of $1 \mathrm{~Hz}$. The day of ADT determination was considered day 0 of kindling. Stimulation was performed daily, and seizure activity after each stimulation was rated according to the criterion of Racine (1972) as modified for the mouse: stage 0, no response or behavior arrest; stage 1, chewing or head nodding; stage 2, chewing and head nodding; stage 3, forelimb clonus; stage 4, bilateral forelimb clonus and rearing; and stage 5, falling. Kindling stimulation was continued until stage 5 seizures were elicited on three consecutive days, which is considered the fully-kindled state. Mice were used even after reaching fully kindled status to test seizure persistence and epileptogenic erasure

Rapid Hippocampus Kindling Model of Epileptogenesis. Rapid kindling is a model of epileptogenesis that allows accelerated evaluation of experimental manipulations during the progression of epilepsy induction (Lothman and Williamson, 1993; Sankar et al., 2010). The rapid kindling procedure was similar to the conventional kindling previously described except that stimulations were applied every 30 minutes until mice exhibited consistent stage 5 seizures. This procedure has been used extensively as a model of compressed epileptogenesis as an alternative paradigm to the conventional kindling models (Sankar et al., 2010; Reddy and Mohan, 2011).

Motor Toxicity Test. Sodium butyrate and other test drugs were evaluated for motor toxicity by using a modification of the horizontal screen test as described previously (Reddy and Jian, 2010). Mice were placed on a horizontally oriented grid (consisting of parallel $1.5-\mathrm{mm}$ diameter rods situated $1 \mathrm{~cm}$ apart), and the grid was inverted. Mice failing to remain on the screen for 60 seconds were considered failures due to excessive sedation or motor ataxia.

Brain Histologic Timm Staining. Timm staining of mossy fiber sprouting is a standard histologic index of epileptogenesis. Timm staining was conducted as described previously (Cavazos et al., 1991; Rao at al., 2006). Mice were deeply anesthetized with ketamine/xylazine mix and transcardially perfused with $75 \mathrm{ml} 0.9 \%$ saline solution followed by $100 \mathrm{ml} 1 \%$ sodium sulfide solution. Perfusion of $100 \mathrm{ml}$ of $4 \%$ paraformaldehyde in $0.1 \mathrm{M}$ phosphate buffer $(7.4 \mathrm{pH})$ followed, and a final perfusion with $50 \mathrm{ml} 1 \%$ sodium sulfide was carried out. The brain was dissected and postfixed in $4 \%$ paraformaldehyde overnight at $4^{\circ} \mathrm{C}$. Brains were then processed with phosphate buffer and sucrose treatment and cut into $20 \mu \mathrm{m}$ transverse sections with a cryostat. Slices were then dry mounted onto slides and allowed to dry overnight. Gum arabic, citrate buffer, hydroquinone, and silver lactate reagents were mixed mechanically on the day of staining. Mounted slides were uniformly soaked in the Timm stain in the dark for 3.5 hours. Slides were then washed with distilled water and counterstained with $0.1 \%$ cresyl violet, if desired. Slides were permanently fixed with DPX Mountant (Sigma, St. Louis, MO) and allowed to dry before imaging and analysis. Staining intensity for Timm histology was quantitatively measured by densitometric analysis. Densitometry was completed in hippocampus regions of interest with Photoshop software (Adobe Systems, San Jose, CA). The mean density of grayscale staining was normalized to the area and white background. Density scores were then nonparametrically graded using a linear scale.

Test Drugs and Treatment Protocols. There are many HDAC inhibitors, such as vorinostat, trichostatin-A, valproic acid, and sodium butyrate (Younus and Reddy, 2017b). Sodium butyrate was the focus of this study for its broad-spectrum HDAC inhibition (Ferrante et al., 2003; Deutsch et al., 2008; Takuma et al., 2014). Valproic acid, a widely used antiepileptic drug, was not used because it is a limited-spectrum HDAC inhibitor and hence offers comparatively limited disease modification of epileptogenesis (Silver et al., 1991; Gurvich et al., 2004; Takuma et al., 2014). The overall experimental protocol for drug treatment and kindling stimulations is illustrated in Fig. 2. In the first kindling development study (protocol 1), sodium butyrate $(600 \mathrm{mg} / \mathrm{kg})$ was injected intraperitoneally twice daily (9 AM and $5 \mathrm{PM}$ ) to maintain chronic HDAC inhibition. The dose of butyrate used in this study has been shown previously to significantly inhibit multiple HDACs and reliably increase the acetylation of H3 and $\mathrm{H} 4$ histones in the brain and other tissues (Ferrante et al., 2003; Takuma et al., 2014). Sodium butyrate has been shown to effectively inhibit downstream $\mathrm{H} 3$ and H4 histone signaling in cells in vitro and after administration in animals (Davie, 2003; Minamiyama et al., 2004). Our design consists of treated animals $(n=11)$ alongside an untreated control group $(n=10)$. Animals were stimulated daily in the morning and received their respective treatments daily until becoming fully kindled. Kindling, however, is a permanent phenomenon and an intense seizure can be elicited weeks or months after kindling development. Therefore, 8 weeks after inducing stage 5 kindling, both groups of mice were tested for persistence of the kindling state by restimulation at the baseline $\mathrm{ADT}$. Additionally, mice were also tested for retention through additional stimulations at 20-minute intervals, increasing current in $25 \mu \mathrm{A}$ increments until the occurrence of stage 4 or 5 seizures. This protocol is henceforth referred to as chronic treatment. In the second kindling development study (protocol 2), sodium butyrate $(600 \mathrm{mg} / \mathrm{kg})$ was injected intraperitoneally twice daily (9 AM and $5 \mathrm{PM}$ ) for exactly 14 days, and animals were stimulated daily even after the end of drug treatment until they became fully kindled. These animals $(n=8)$ were treated alongside animals $(n=9)$ receiving the synthetic neurosteroid ganaxolone [(GX), $3 \beta$-methyl-3 $\alpha$-hydroxy-5 $\alpha$-pregnan-20-one] $(0.5$ or $1.0 \mathrm{mg} / \mathrm{kg}$ ) 15 minutes prior to stimulation for 14 days, as well as a combination group $(n=9)$ receiving butyrate twice daily and GX 15 minutes prior to stimulation. GX is an allosteric modulator of the $\mathrm{GABA}_{\mathrm{A}}$ receptor with antiseizure properties (Reddy and Rogawski, 2010). This protocol is henceforth referred to as subchronic treatment. The final kindling study format (protocol 3) involved two groups of animals stimulated daily without any drug treatment until achieving complete kindling. To test the retroactive effect of epigenetic treatment on epilepsysusceptible mice, sodium butyrate $(600 \mathrm{mg} / \mathrm{kg}$, i.p.) was injected twice daily in the experimental group $(n=17)$ for 2 weeks, during which time no stimulations were conducted. A control group $(n=12)$ received no treatment during this time. At the end of the treatment period, ADTs were redetermined for all animals and all mice were rekindled until they exhibited consistent stage 5 seizures. In another test, as a measure of the drugs' acute seizure suppression, butyrate and/or GX were also administered as one-time pretreatments to these previously kindled mice. In parallel studies, we tested the effect of the synthetic neurosteroid GX on kindling progression. GX is an effective antiseizure agent in the kindling model (Reddy and Rogawski, 2010). Agematched male mice $(n=7-10)$ were given sodium butyrate (300 or $600 \mathrm{mg} / \mathrm{kg}$, i.p.) approximately 2 hours before stimulation, GX ( 0.5 or $1.0 \mathrm{mg} / \mathrm{kg}$, s.c.) 15 minutes before stimulation, or both butyrate $(600 \mathrm{mg} / \mathrm{kg})$ and $\mathrm{GX}(0.5 \mathrm{mg} / \mathrm{kg})$ before stimulation. 

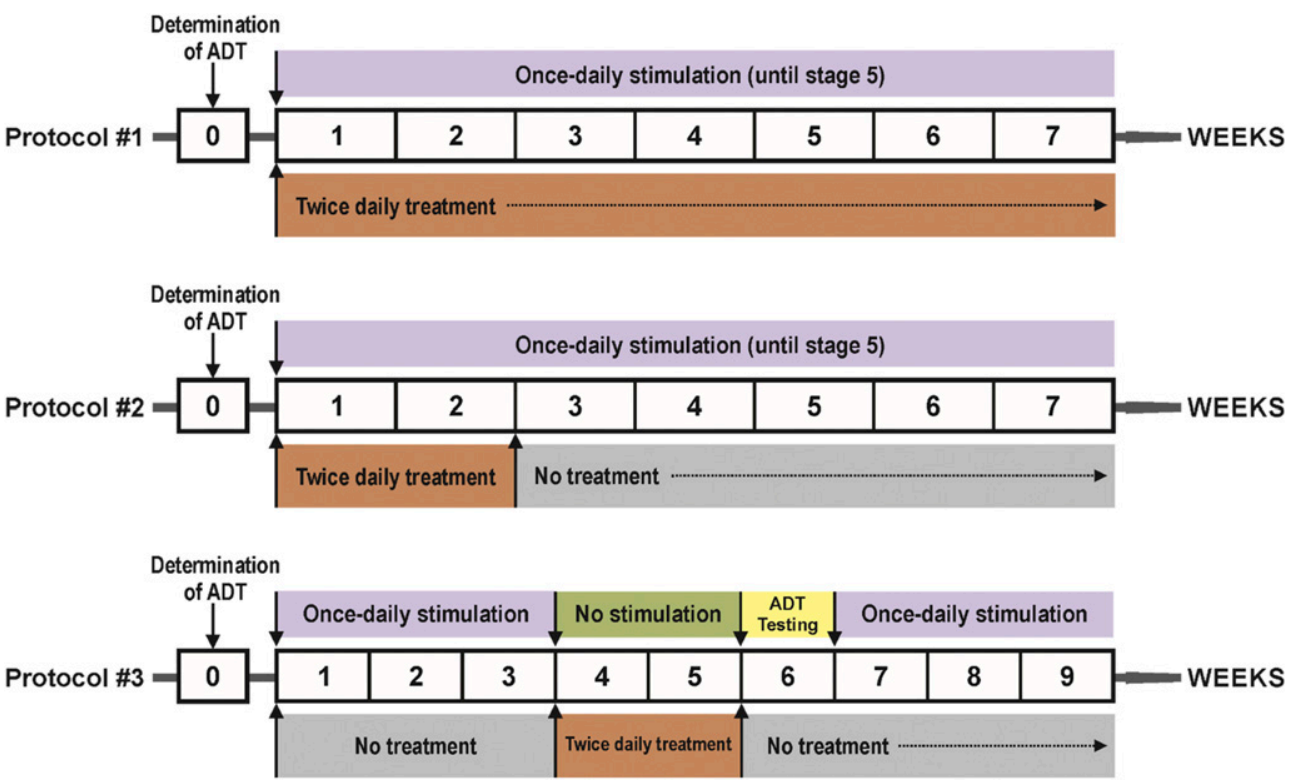

Fig. 2. Overall experimental design. In protocol 1, animals were treated with butyrate $(600 \mathrm{mg} / \mathrm{kg}$, i.p., twice daily) until they achieved the fully kindled state, exhibiting consistent stage 5 seizures on three consecutive days. In protocol 2 , animals were treated with butyrate $(600 \mathrm{mg} / \mathrm{kg}$ i.p., twice daily), GX $(0.5 \mathrm{mg} / \mathrm{kg}$, s.c., 15 minutes prior to stimulation), or a combination of both drugs for 2 weeks, followed by a drug-free withdrawal period until fully kindled. In protocol 3, animals were fully kindled without any injected treatment, followed by a 2 -week period of butyrate treatment $(600 \mathrm{mg} / \mathrm{kg}$, i.p., twice daily) and hiatus from stimulation. On the sixth week, treatment was discontinued and individual ADTs were redetermined. Animals were subsequently rekindled to full without treatment, identical in procedure to the initial kindling.
Data Analysis. Group data are expressed as the mean \pm S.E.M. Differences in kindling seizure stages between groups were compared with the nonparametric Kruskal-Wallis test followed by the MannWhitney $U$ test. Comparison of the mean values of the $\mathrm{AD}$ duration between groups was made with one-way analysis of variance, followed by an unpaired two-tailed Student's $t$ test. Comparison of the percentage of animals exhibiting seizures and the percentage difference in seizure duration in various groups was made by the Wilcoxon signed ranks test. In all statistical tests, the criterion for statistical significance was $P<0.05$.

\section{Results}

Sodium Butyrate Inhibits HDAC Activity in the Brain. To determine the ability of sodium butyrate to inhibit HDAC activity, we performed HDAC activity assay in hippocampus and cortex samples from untreated control and butyrate-treated $(600 \mathrm{mg} / \mathrm{kg}$, i.p.) mice. HDAC activity assay was carried out by the Fluor-de-Lys HDAC activity assay kit, as described previously (Nian et al., 2009; Rajendran et al., 2011). Our results showed that HDAC activity in the hippocampus and cortex of mice treated with sodium butyrate was significantly $(20 \%-40 \%, P<0.05)$ reduced compared with that of control mice. Hippocampal HDAC activity in the butyrate group $(1.0 \pm 0.02 \mathrm{HDAC} \%$ control after normalization per microgram protein) was significantly lower than in the control group $(1.2 \pm 0.06)$. Similarly, sodium butyrate significantly reduced HDAC activity in the cortex $(1.1 \pm 0.01 \mathrm{HDAC} \%$ control after normalization per microgram protein) compared with the control group $(1.5 \pm 0.16)$. These results confirm the significant inhibition of HDAC activity in mice following sodium butyrate treatment, as consistent with earlier reports (Ferrante et al., 2003; Takuma et al., 2014).

Acute HDAC Inhibition Produces Little Change in Seizure Expression in Fully Kindled Mice. To determine the acute effect of HDAC inhibition by sodium butyrate on kindled seizures, we tested butyrate in a dose-dependent fashion in fully kindled mice expressing stage 5 (generalized) seizures. The acute effect of sodium butyrate is shown in
Fig. 3. Sodium butyrate pretreatment 1 hour before stimulation in fully kindled animals provided little or no seizure protection, as evident from the lack of seizure suppression by either dose of butyrate (Fig. 3A). Butyrate also did not significantly affect the mean $\mathrm{AD}$ duration (Fig. 3B). To determine the comparative and combination efficacy, we also tested the neurosteroid antiseizure agent GX (Reddy and Rogawski, 2010). Our results showed that while neither a low dose of GX $(0.5 \mathrm{mg} / \mathrm{kg})$ nor a combination treatment of butyrate and GX provided acute protection, a high dose of GX $(1.0 \mathrm{mg} / \mathrm{kg})$ displayed modest seizure protection (Fig. 3D). None of these treatments, however, significantly influenced $\mathrm{AD}$ duration (Fig. 3E). An important criterion to take into consideration when determining dose selection, however, is the significant animal behavioral sedation resulting from high dosage, which is not as prevalent in the selected doses of butyrate or GX (Fig. 3, C and F). In addition, we tested the effect of sodium butyrate on acute seizures at longer intervals between treatment and stimulation. Similar seizure outcomes resulted even 4 hours after treatment, since all animals exhibited stage $4 / 5$ seizures ( $n=9$ /group). These results indicate that acute treatment with butyrate has no effect on seizure expression and motor behavior.

Chronic HDAC Inhibition by Sodium Butyrate Inhibits the Development of Limbic Epileptogenesis. To determine the chronic effect of HDAC inhibition by sodium butyrate on kindled epileptogenesis progression, we administered butyrate twice daily to several animals, which were concurrently kindled with a control group (Fig. 2). The development of kindled seizures in control mice and mice treated daily with sodium butyrate $(600 \mathrm{mg} / \mathrm{kg})$ is shown in Fig. 4 . There was no significant difference in mean ADT between the control and butyrate groups (Fig. 4B). Sodium butyrate produced marked impairment in the development of kindling epileptogenesis, most evident in the significant suppression of seizures at corresponding stimulation sessions compared with the control (Fig. 4C). Mean AD duration, however, was not significantly reduced (Fig. 4D). Mice treated with butyrate also showed a drastically decreased rate of kindling, measured 
A

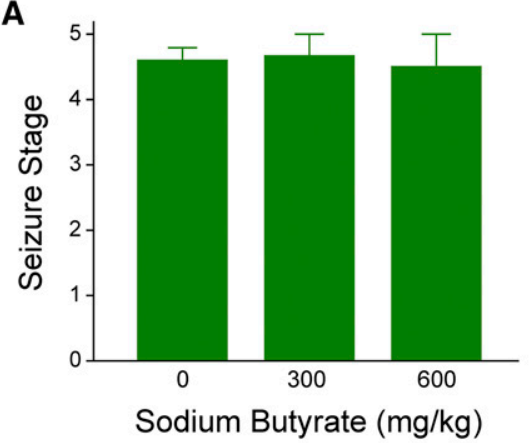

B

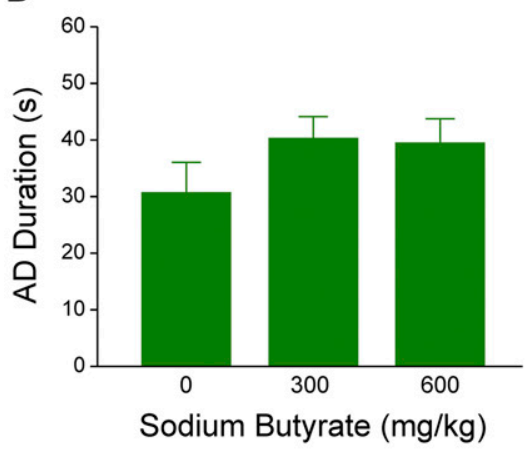

C

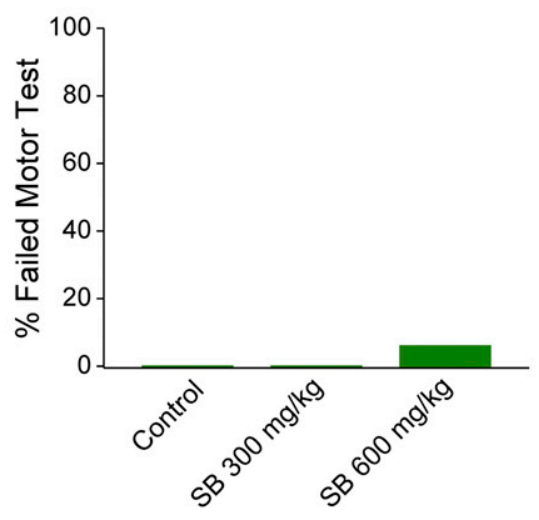

D
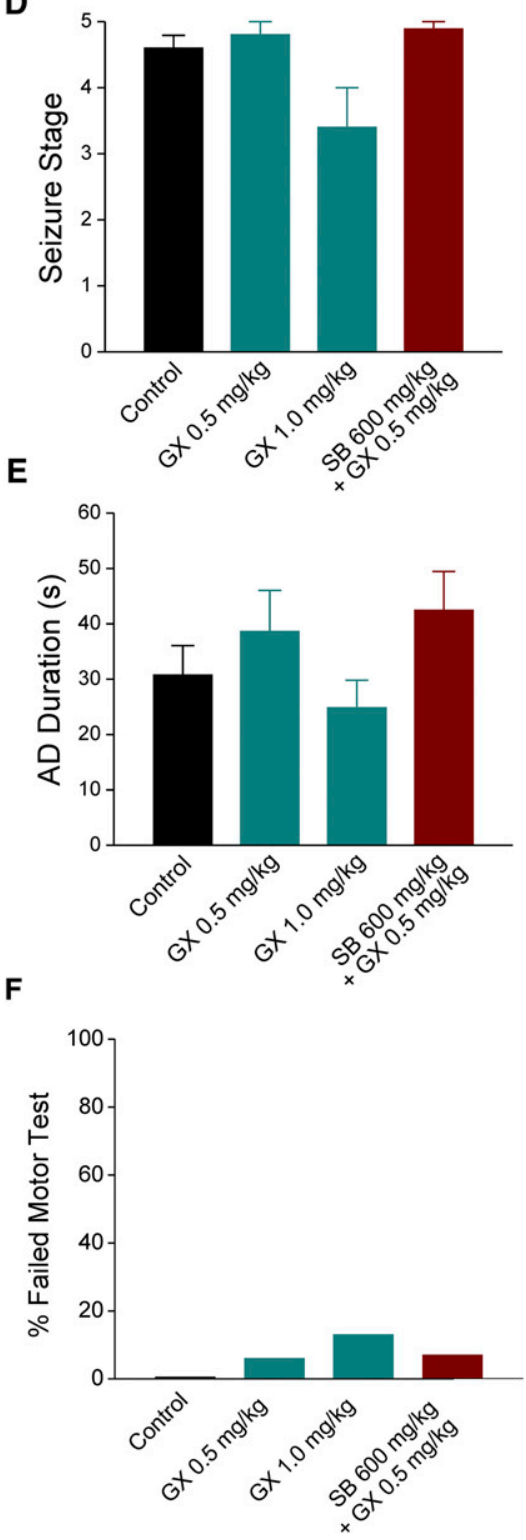

Fig. 3. HDAC inhibition by butyrate produces little acute seizure suppression and motor activity in epileptic mice. (A) Sodium butyrate given 1 hour before stimulation in fully kindled mice fails to reduce severity of acute seizure expression. (B) $\mathrm{AD}$ duration was similar between treated and control groups. (C) Limited effect of sodium butyrate on motor ataxia. Mice were allowed to climb on an inverted screen, and time before falling was monitored. Animals failing to remain on the screen for at least 60 seconds were considered failures due to sedation or motor ataxia. (D) Slight acute effect of GX (0.5 and $1 \mathrm{mg} / \mathrm{kg}$, s.c.) and/or butyrate on behavioral seizure expression in fully kindled mice. (E) None of the treatment groups displayed a significant change in $\mathrm{AD}$ duration. (F) Mild effect of GX on motor ataxia in mice. All values represent the mean \pm S.E.M. ( $n=9-15$ mice/group). by the number of stimulations required to elicit three consecutive stage 5 (generalized) seizures, in comparison with the control group (Fig. 4E; Table 1). These results indicate the striking epileptogenesis-delaying effect of persistent HDAC inhibition by butyrate treatment.

Chronic HDAC Inhibition by Sodium Butyrate Impairs Lasting Retention of Limbic Epileptogenesis. To examine the long-term antiepileptogenic inhibition of sodium butyrate, both groups of animals were restimulated at their baseline ADT 8 weeks after the initial chronic kindling to determine the strength of epileptogenic retention (Fig. 5). While the majority of control animals still displayed severe generalized seizures (stage 4 or 5), only a slight minority of animals treated with butyrate in the initial study showed such seizures, demonstrating butyrate's prolonged diseasemodifying effect even without drug administration (Fig. 5A). Furthermore, during ADT redeterminations (Fig. 5B), butyrate-treated animals displayed significant seizure resistance compared with control animals at corresponding stimulation currents, and this butyrate group required $50 \mu \mathrm{A}$ more current (a 33\% increase) than the control group to achieve stage 5 seizures. Butyrate did not significantly affect the mean $\mathrm{AD}$ duration in this retention study (Fig. 5C). These results indicate that HDAC inhibition by sodium butyrate carries a long-lasting antiepileptogenic or diseasemodification effect.

Subchronic HDAC Inhibition by Sodium Butyrate Inhibits the Development of Limbic Epileptogenesis. To observe the effects of sodium butyrate treatment and discontinuation within the same kindling cycle, we administered sodium butyrate to various subgroups, alongside a control group, for 2 weeks, and then allowed kindling to continue without treatment (Fig. 2). The development of kindled seizures in control mice and mice treated for 14 days with sodium butyrate $(600 \mathrm{mg} / \mathrm{kg})$ is shown in Fig. 6 . There was no significant difference in mean ADT between any of the four groups in the study (Fig. 6E). During the 14 days of injection, sodium butyrate strikingly inhibited the 
A

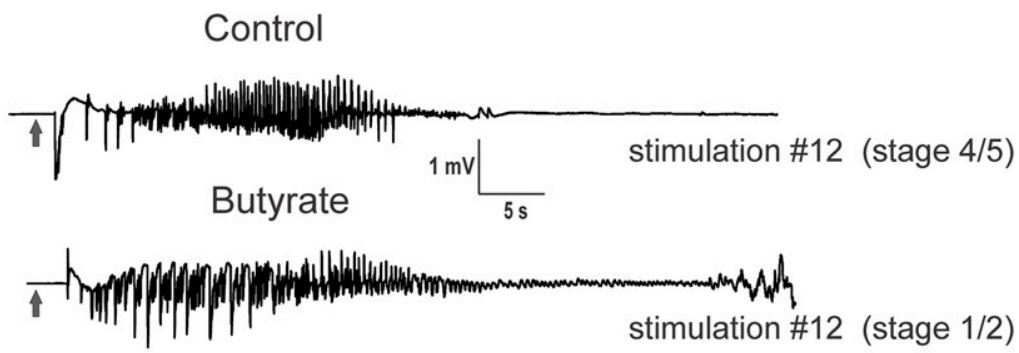

C

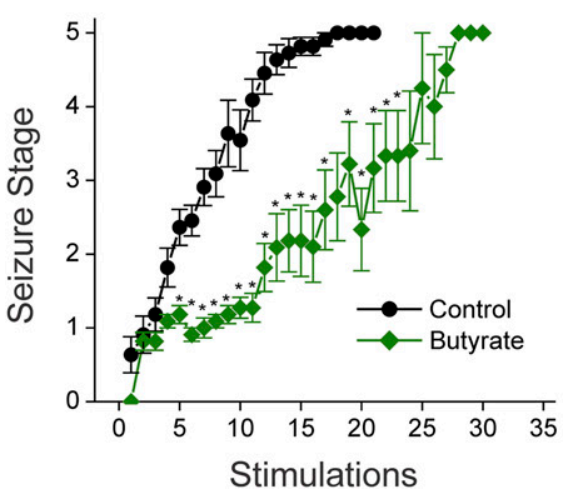

D

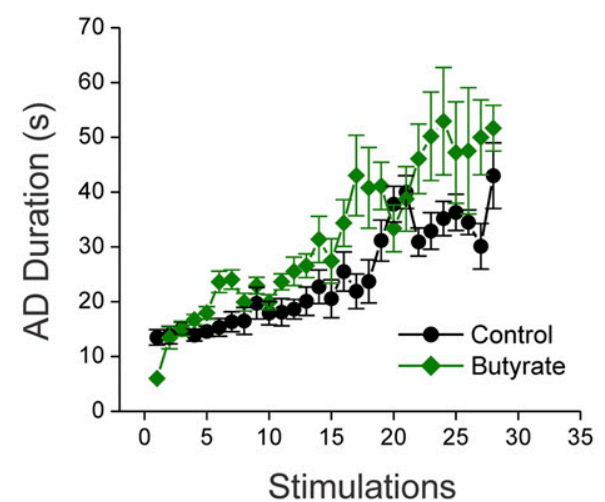

B

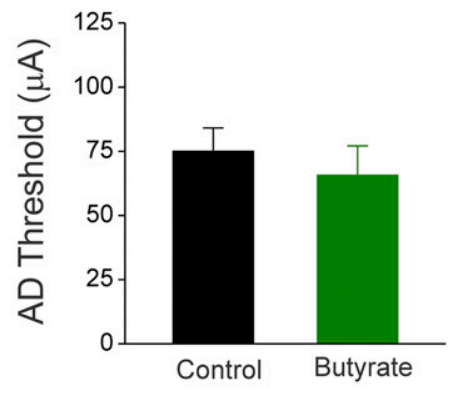

E

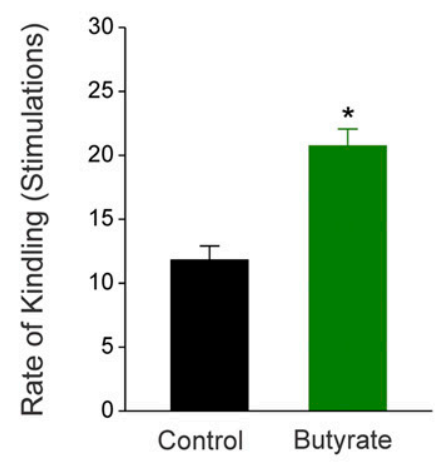

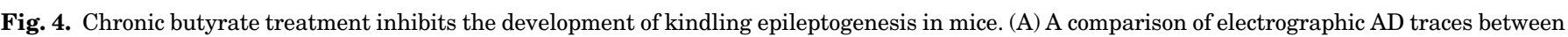

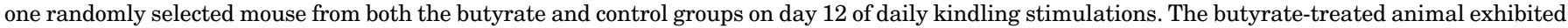

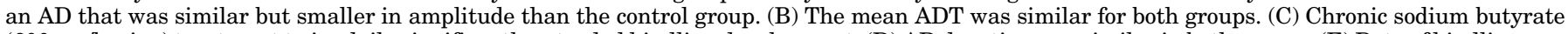

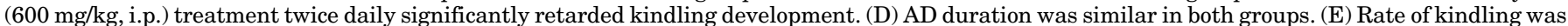

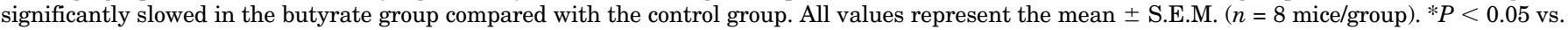
control group.

development of kindling epileptogenesis (Fig. 7), seen prominently in the significantly reduced seizure stages at corresponding stimulation sessions compared with the control (Fig. 6A). Butyrate, however, did not significantly affect the mean $\mathrm{AD}$ duration overall (Fig. 6B). Consistent with the chronic group (Fig. 4), subchronic butyrate treatment led to complete retardation of kindling epileptogenesis progression beyond stage 2/3 (partial) seizures despite daily stimulation for 35 days (Fig. 6, A and $\mathrm{B}$; Table 1), which indicates a true antiepileptogenic effect. To determine whether inhibition of HDAC confers synergistic effects when used in combination with an antiseizure drug, we studied the effect of we have already defined GX many times previously GX alone or in combination with butyrate. Treatments of GX $(0.5 \mathrm{mg} / \mathrm{kg})$, as well as the combination treatment of GX $(0.5 \mathrm{mg} / \mathrm{kg})$ and butyrate $(600 \mathrm{mg} / \mathrm{kg})$, induced similar levels of epileptogenic retardation compared with the butyrate-alone group (Fig. 4C). Similarly, these treatments also did not significantly affect the mean $\mathrm{AD}$ duration (Fig. 6D). Expectedly, the antiepileptogenic butyrate and/or GX treatments significantly retarded the development of kindled seizures compared with the control (Fig. 6F), indicating modest synergistic protection offered by the antiseizure GX.

Acute HDAC Inhibition Fails to Affect EpileptogenicLike Response in Rapid Kindling Epileptogenesis. To further elucidate the effective window of HDAC mechanisms in susceptibility to epileptogenesis (acute vs. chronic inhibition), we used the rapid kindling model of acute epileptogenesis, the process of epilepsy induction by stimulation every 30 minutes (acute), rather than the once-daily (chronic) stimulation implemented in the classic kindling model. Acute treatment with butyrate did not modify the rapid kindling progression, as evident by the essentially identical seizure stage and $\mathrm{AD}$ duration curves of control and butyrate groups (Fig. 8, A-D). A representative electroencephalogram discharge is shown in Fig. 8E. There was no significant difference in mean ADT between the control and butyrate groups (Fig. 8C). Furthermore, butyrate did not significantly affect the rate of kindling compared with the control, indicating the inability of butyrate treatment for acute HDAC inhibition to inhibit rapid epileptogenic

\section{TABLE 1}

Mean number of stimulations to achieve kindling stages in vehicle-treated control and butyrate-treated mice

Values represent mean \pm S.E.M. of number of stimulation values derived from the experiments in Figs. 5C, 6A, and 8C.

\begin{tabular}{lrcc}
\hline & \multirow{2}{c}{ Vehicle } & \multicolumn{2}{c}{ Butyrate Treatment } \\
\cline { 3 - 4 } & & \multicolumn{1}{c}{ Chronic } & \multicolumn{1}{c}{ Subchronic } \\
\hline Stage 1 & $1.9 \pm 0.31$ & $2.3 \pm 0.19$ & $1.4 \pm 0.26$ \\
Stage 2 & $3.4 \pm 0.51$ & $14.2 \pm 1.54^{*}$ & $15.3 \pm 2.51^{*}$ \\
Stage 3 & $7.5 \pm 0.68$ & $15.8 \pm 1.71^{*}$ & $16.7 \pm 2.43^{*}$ \\
Stage 4 & $10.3 \pm 0.89$ & $18.4 \pm 1.40^{*}$ & $22.5 \pm 3.22^{*}$ \\
Stage 5 & $12.3 \pm 0.92$ & $20.3 \pm 1.47^{*}$ & $23.5 \pm 3.30^{*}$ \\
\hline
\end{tabular}

$* P<0.05$ vs. vehicle control. 
A

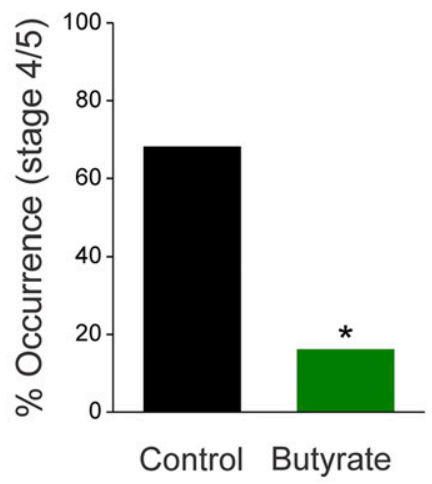

B

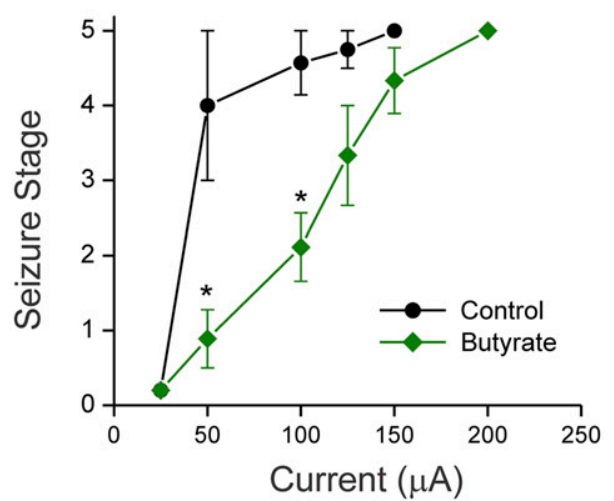

C

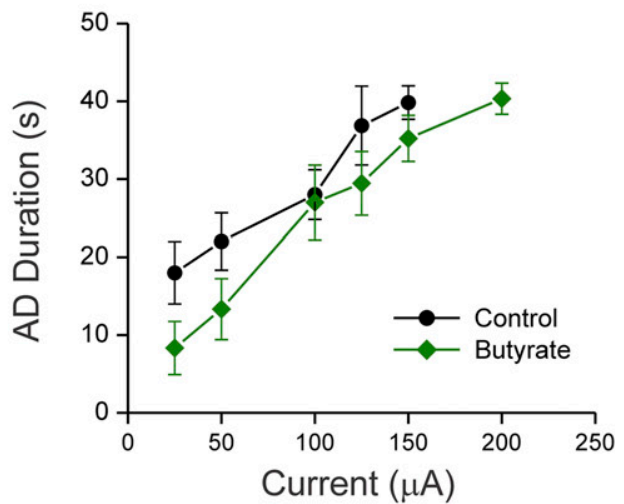

Fig. 5. Butyrate treatment shows prolonged disease modification long after initial treatment in epileptic mice. (A) 8 weeks after the original study in Fig. 2, animals of both groups were re-stimulated at their past ADT values. Animals from the sodium butyrate cohort displayed marked resistance for induction of generalized seizures. (B) Animals of both groups were subjected to increased stimulation currents until they elicited stage 5 seizures. Animals previously treated with butyrate required significantly greater currents to display the corresponding stage seizures compared with the control. (C) Animals from the butyrate cohort displayed reduced $\mathrm{AD}$ durations during initial stimulations. All values represent the mean \pm S.E.M. $(n=6-8$ mice/group). $* P<0.05$ vs. control group.

development (Fig. 8D). These results are consistent with the evidently delayed effects of epigenetic interruption of epileptogenesis.

HDAC Inhibition Reverses Epileptogenic State in Fully Kindled Epileptic Animals. To determine any disease-modification effects of HDAC inhibition in animals that have already developed advanced stages of epilepsy, we administered butyrate in previously fully kindled animals with stage 5 (generalized) seizures to check epileptogenic reversal or erasure of epileptic state. Animals were fully kindled without any injected treatment until they showed consistent stage 5 seizures, followed by a 2 -week period of
A

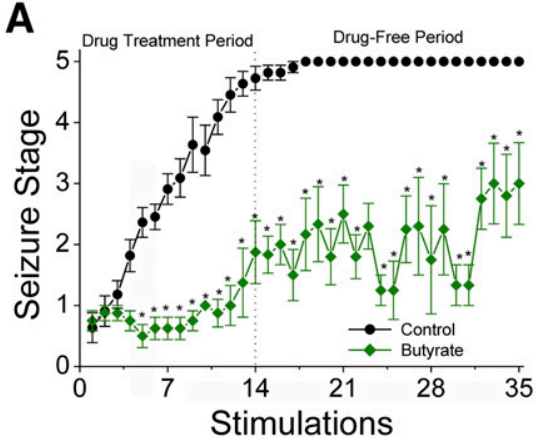

B

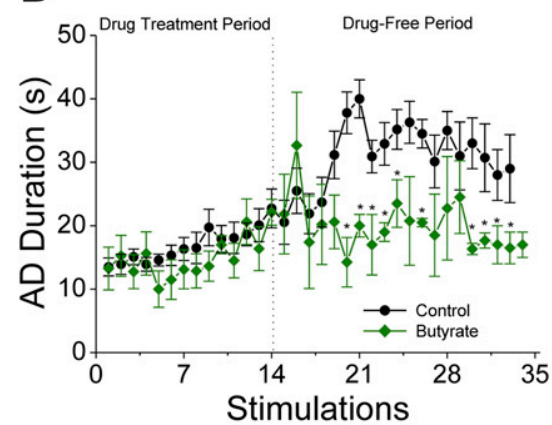

C

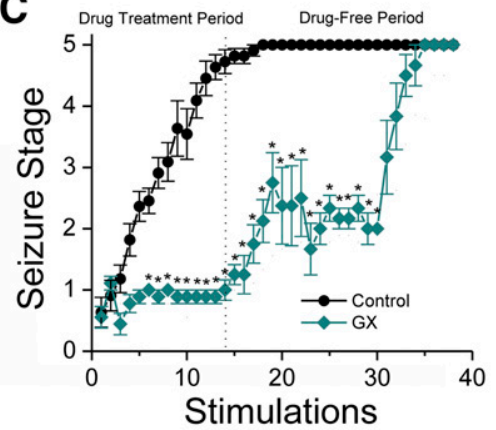

D

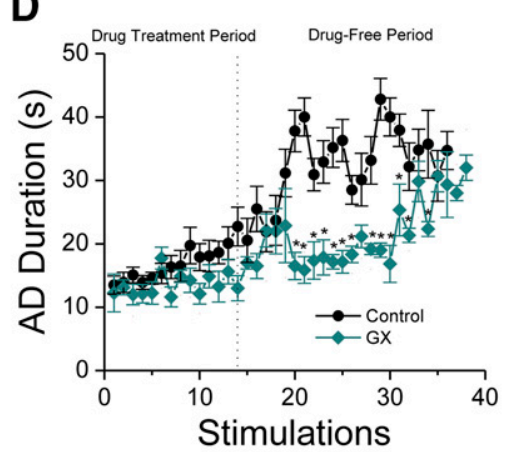

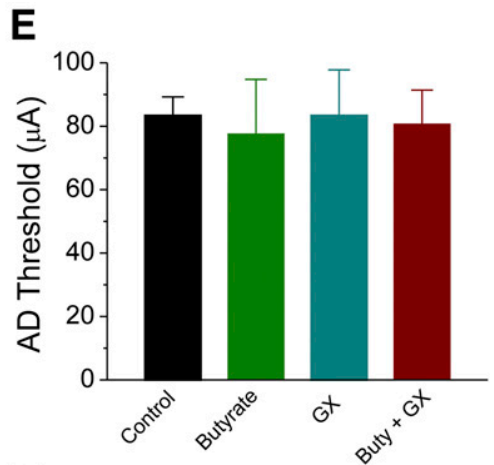

$\mathbf{F}$

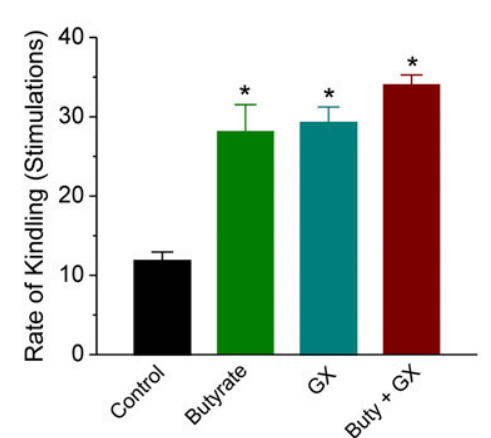

Fig. 6. Subchronic butyrate treatment inhibits the development of kindling epileptogenesis in mice. (A) Sodium butyrate (600 mg/kg, i.p.) treatment twice daily for 14 days (dotted line) greatly retarded kindling development. This antiepileptogenic effect persisted even during the drug-free period. (B) $\mathrm{AD}$ duration was similar in both groups for the first 17 days, after which butyrate-treated animals demonstrated notably shortened $\mathrm{AD}$ durations. (C) Subchronic GX $(0.5 \mathrm{mg} / \mathrm{kg}$, s.c.) 15 minutes before stimulation markedly slowed kindling development. At the beginning of the drug-free period, however, mice treated with GX displayed a sharp increase in seizure severity. (D) The GX group did not show significant reduction in mean AD duration in comparison with the control group for the first 18 days, but displayed significant $\mathrm{AD}$ duration reduction in the remaining days. (E) The mean ADT was similar for the control, butyrate-only, GX-only, and GX-butyrate combination groups. (F) Rate of kindling was significantly delayed in all treated groups compared with the control group. For butyrate-treated animals that failed to show stage $4 / 5$ seizures, a cutoff value of 35 was assigned for comparative analysis. All values represent the mean \pm S.E.M. $\left(n=9\right.$ mice/group). ${ }^{*} P<0.05$ vs. control group. 


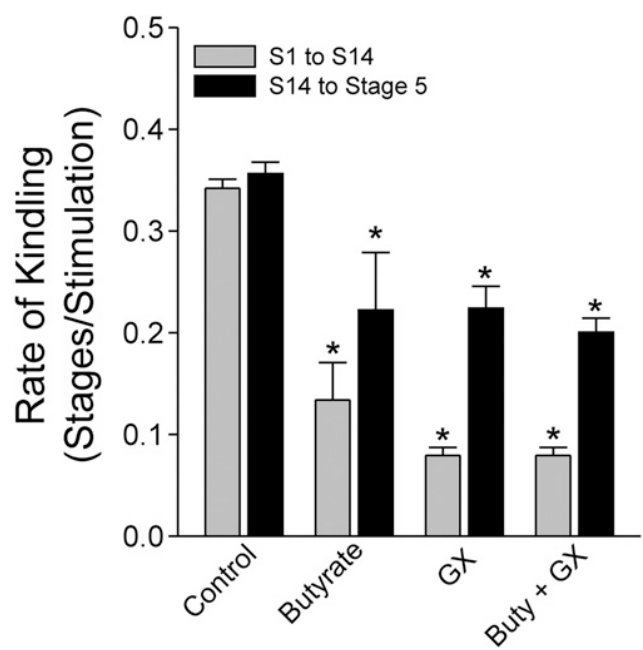

Fig. 7. Comparison of the rate of kindling developing during subchronic treatment and following withdrawal period in mice. Mean seizure stage values from stimulations 1 to 14 or stimulation 14 to stage 5 kindling (Fig. 6 , A and C) were fit to the linear function $S_{n}=R_{n}+A$, where $S_{n}$ is the mean seizure stage value for the $n$th stimulation, $R$ is the rate of kindling, and $A$ is set equal to either 0 (stimulations $1-14$ ) or $S_{14}$ (stimulation 14 to stage 5 kindling). The rate of kindling $R_{n}$ for each of the four groups is depicted above. The control group exhibited no significant kindling difference before and after the 2-week cutoff, while all treatment groups demonstrated drastically increased rate of kindling following withdrawal of treatment, although this was still slower in rate than that of the control group. Values represent the mean \pm S.E.M. $* P<0.05$ vs. control group.

butyrate treatment $(600 \mathrm{mg} / \mathrm{kg}$, i.p., twice daily) and hiatus from stimulation (Fig. 2). In the sixth week, treatment was discontinued and individual ADTs were redetermined. Animals were subsequently rekindled to full without treatment, identical in procedure to the initial kindling. Butyrate-treated mice displayed a significant reduction in seizure severity when tested the week following termination of butyrate therapy (Fig. 9A). During rekindling, butyrate-treated mice required significantly more stimulations to rekindle fully (Fig. 9, A and C). Furthermore, $\mathrm{AD}$ duration among treated mice progressively increased, while $\mathrm{AD}$ durations of control mice remained relatively constant across stimulations (Fig. 9B). Thus, these results indicate that HDAC inhibition by butyrate treatment can promote significant unwiring or erasure of even prior chronic epileptogenic state (curing epilepsy).

HDAC Inhibition Prevents Epileptogenic Mossy Fiber Axonal Sprouting in the Hippocampus. To determine whether HDAC inhibition by sodium butyrate treatment is associated with morphologic changes in neuronal circuits involved in epileptogenesis, we examined the extent of mossy fiber sprouting in hippocampus samples isolated from control and butyrate-treated animals. The pathologic role of mossy fiber sprouting in temporal lobe epileptogenesis is controversial because inhibition of mossy fiber sprouting may not reflect an antiepileptogenic process (Heng et al., 2013). Nevertheless, mossy fiber sprouting is the most commonly used morphologic marker of epileptogenesis in experimental models and it provides valuable information regarding the impact of therapeutic interventions on aberrant sprouting of granule cell axons in the dentate gyrus. A characteristic histologic sign of TLE is the loss of hilar cells in the dentate gyrus and mossy fiber sprouting. Mossy cells in the hippocampus are highly excitable. Mossy cells are bidirectionally connected through a positive feedback loop to granule cells, the principal cells of the dentate gyrus. This recurrent circuit is strategically placed between the entorhinal cortex and the CA3 region, and is thus potentially proconvulsive and may promote epileptogenesis (Ratzliff et al., 2002). We observed a significant increase in mossy fiber sprouting in control, fully kindled animals (Fig. 10A). Quantification of axonal sprouting within the dentate gyrus region by densitometry and staining intensity scoring revealed complete reversal of seizure-induced increase in mossy fiber sprouting in animals treated with butyrate (Fig. 10, B-E), suggesting possible disease modification from HDAC inhibition. Butyrate, when given in combination with GX, also resulted in significant reduction in mossy fiber sprouting (Fig. 10, A and E), indicating further disease-modifying potential of the antiseizure treatment with GX.

\section{Discussion}

The present study shows that targeted subchronic and chronic inhibition of HDACs can inhibit or stop the progression of epileptogenesis in experimental models of TLE. The key observations from this study are the following: 1) daily treatment with the HDAC inhibitor sodium butyrate significantly retarded the development of kindling epileptogenesis without affecting $\mathrm{AD}$ activity; 2) the persistence of this epileptic condition was also impaired in butyrate-treated animals; 3) subchronic butyrate treatment of 2 weeks led to strong retardation of epileptogenesis progression; 4) butyrate treatment carried long-term antiepileptogenic retention, whether administered during or after kindling; 5) butyrate treatment has little or no behavioral motor toxicity; 6) acute butyrate treatment did not affect rapid kindling epileptogenesis; 7) butyrate treatment facilitated a strong reduction in mossy fiber sprouting; and 8) a combination treatment of butyrate and an antiseizure neurosteroid showed further disease modification of epileptogenesis. Collectively, these results indicate that the HDAC signaling pathway mediates the development and persistence of epileptogenesis in an experimental model of TLE.

Epigenetic HDAC Signaling in Epilepsy. There is emerging evidence that epigenetic signaling plays a crucial role in the pathophysiology of epileptogenesis and chronic epilepsy (Qureshi and Mehler, 2010a; Kobow and Blümcke, 2011; Younus and Reddy, 2017b). The main epigenetic mechanisms include histone modifications, DNA methylation, and microRNA-based transcriptional control (Fig. 1). The primary histone modification associated with epilepsy and other neural disorders is lysine acetylation at the histone tail (Kobow and Blümcke, 2011). Histone acetylation is catalyzed by histone acetyltransferases and histone deacetylation is carried out by HDACs. As a result of these two opposing epigenetic modifications, chromatin is known to exist in two higher-order structures. Euchromatin refers to the loosely packaged and open form that is transcriptionally active, whereas heterochromatin refers to the highly compact and transcriptionally inactive form (Kouzarides, 2007). HDACs play a crucial role in repressing gene transcription by condensing chromatin structure. Removal of the acetyl group increases the positive charge of the histone and confers a more compact state, characteristic of gene repression. HDACs can also remove acetyl moieties from transcription factors, 
A

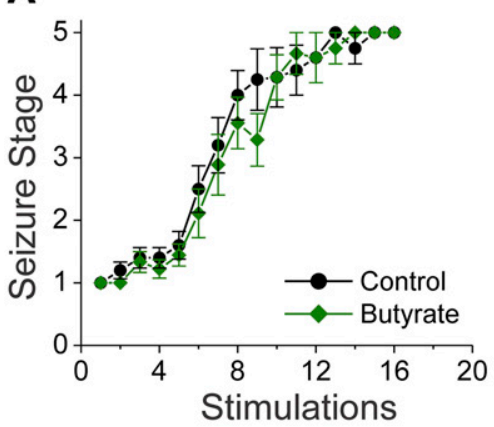

C

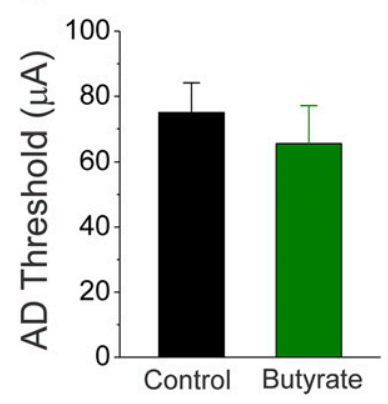

B

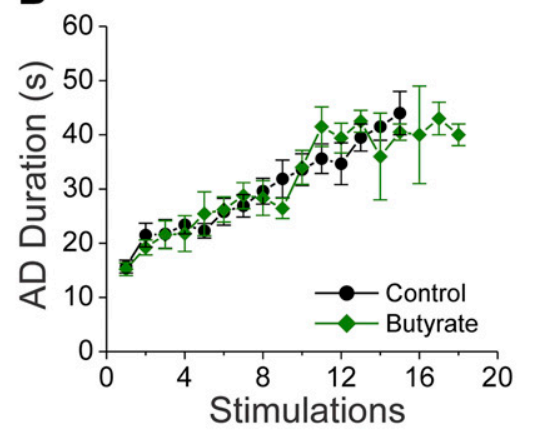

D

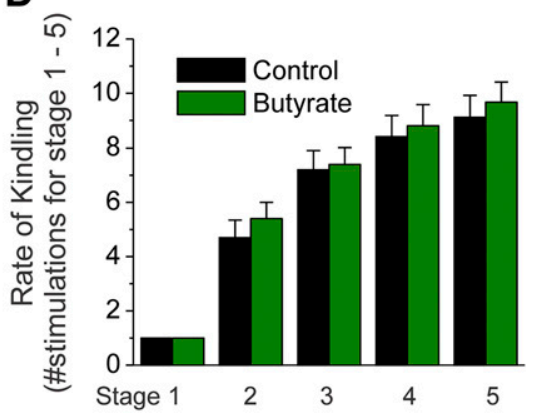

Fig. 8. Acute butyrate treatment fails to affect rapid kindling epileptogenesis in mice. (A) Butyrate (600 mg/kg, i.p.) was given 1 hour prior to stimulation sessions in the designated group. Butyrate-treated mice failed to display any retardation of rapid kindling development. (B) AD duration was also similar in both groups. (C) ADT was similar in both groups. (D) The rate of rapid kindling development, expressed as mean number of stimulations to achieve kindling stage 1-5 seizures, was similar in both groups. (E) A comparison of electrographic $\mathrm{AD}$ activity between one randomly selected mouse from both the butyrate and control groups on stimulation 8 showing similar duration and stage $2 / 3$ seizures. Values represent the mean \pm S.E.M. $(n=6-9$ mice/group).
E

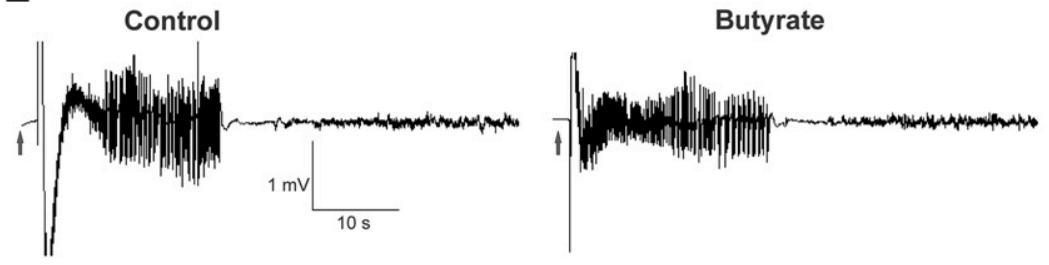

thereby further suppressing gene activity (Morris et al., 2010). There are four classes of HDAC superfamilies, with classes 1 and 2 being the most relevant to epilepsy because they have the highest expression seen in the brain (Gray and Ekström, 2001; Carey and La Thangue, 2006). Thus, HDAC inhibitors are important tools for unraveling the pathology of epilepsy. There are many HDAC inhibitors, such as vorinostat, trichostatin-A, entinostat, and sodium butyrate (Ververis et al., 2013). Sodium butyrate is widely used for broad-spectrum HDAC inhibition and consequent augmentation of H3 and H4 acetylation in the brain (Ferrante et al., 2003; Deutsch et al., 2008; Takuma et al., 2014). In the present study, we presented convincing evidence that chronic but not acute sodium butyrate administration inhibited development of seizures in the kindling model of TLE. These findings suggest that the epigenetic HDAC pathway represents a novel target for the development of new drug disease-modifying capacity for the treatment and prevention of epilepsy. The caveat remains, however, that the exhibited effects of sodium butyrate are unrelated to HDAC inhibition. For example, sodium butyrate is a short-chain fatty acid used as an energy source and directly stimulates NF- $\kappa$ B transcription (Borthakur et al., 2008). However, this is highly unlikely given the significant mechanistic data on HDAC inhibition as assessed by HDAC activity in brain samples. Moreover, there is little evidence of $\mathrm{NF}-\kappa \mathrm{B}$ involvement in seizure susceptibility.
Subchronic and Chronic HDAC Inhibition Retards Epileptogenesis. In the present study, sodium butyrate treatment inhibited HDAC activity but produced no acute antiseizure effect in epileptic animals. Similarly, butyrate treatment was ineffective in reducing the development of rapid kindling. These outcomes were expected given the longer time necessary for the HDAC inhibitor to affect enough gene networks and synaptic processes to manifest in significant protection. During chronic treatment, butyrate significantly hindered the rate of kindling epileptogenesis without affecting $\mathrm{AD}$ progression. The extent of protection, however, was similar to that of the antiseizure GX treatment given 15 minutes before each stimulation. Although both treatments produced similar protective effects against epileptogenesis, sodium butyrate showed exceptionally long-lasting protection (disease modification), whereas GX only temporarily suppressed the seizure occurrence. To delineate the difference between the modes of action of HDAC inhibition, we also performed a subchronic study, wherein animals received butyrate for 2 weeks only, but continued to get stimulations until they reached the epileptogenic state. During the treatment window, both butyrate and GX provided significant hindrance of the kindling process. When treatment was discontinued, however, epileptogenic progression rapidly rose in GX-only groups, while all butyrate-treated groups saw chronic seizure protection even after treatment was 


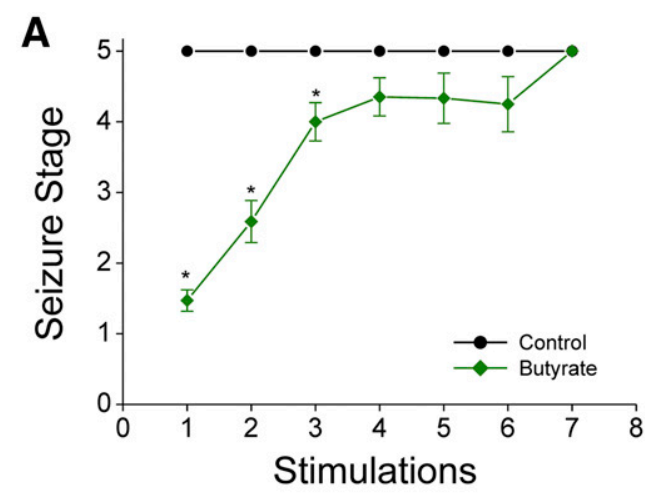

B

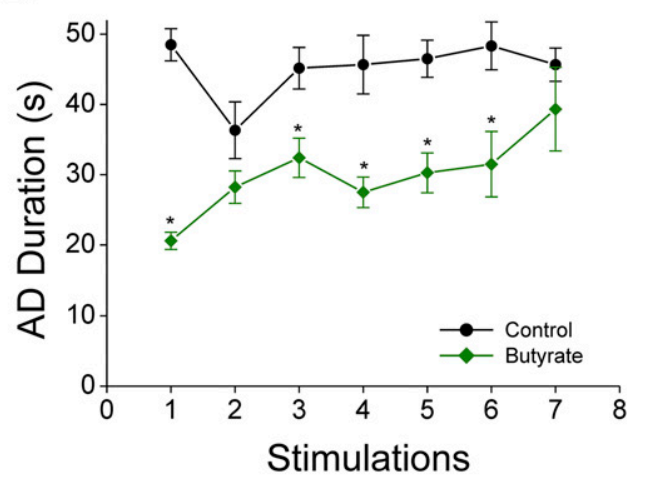

C

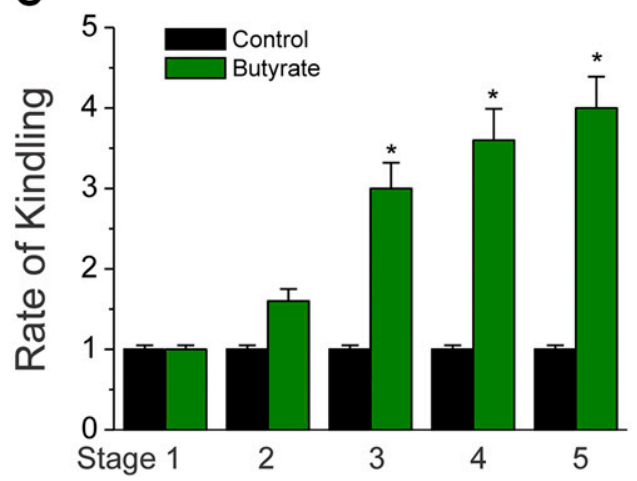

Fig. 9. Fully kindled epileptic animals treated with butyrate exhibit kindling reversal (curing epilepsy). Previously kindled mice expressing stage 4/5 (generalized) seizures were given daily butyrate treatment of 2 weeks. At the end of the treatment period, animals were rekindled to full. (A) Butyrate-treated mice displayed significant reduction in seizure severity during rekindling, in addition to requiring significantly greater stimulations to fully kindle again. (B) In contrast to the relatively steady $\mathrm{AD}$ durations shown in control animals, butyrate-treated mice exhibited progressively longer AD durations with further stimulations. (C) Although both groups required a similar number of stimulations to reach stage 1 and 2 seizures, butyrate-treated animals required significantly more stimulations to reach seizures of stages 3,4 , and 5 . ${ }^{*} P<0.05 \mathrm{vs}$. control group.

discontinued. These results suggest that the induction and persistence of limbic epileptogenesis are markedly reduced by HDAC activity inhibition by butyrate. Focal electrographic $\mathrm{AD}$ is required for induction of epileptogenesis in the kindling model (Goddard et al., 1969; Albertson et al., 1984). The delayed kindling development after HDAC inhibition is not due to differences in focal electrographic seizures because the duration of evoked $\mathrm{AD}$ was similar among control and butyrate groups during the early stimulation session.
However, the progressive increase in duration of the $\mathrm{AD}$ activity with successive stimulations was significantly attenuated in the butyrate group. Since butyrate did not affect rapid kindling progression, the structural and functional circuits that are required to exhibit behavioral and electrographic seizures are not affected by acute HDAC inhibition. Thus, the complex plasticity that is required to transform a normal brain into an epileptic brain in the kindling model is attenuated by chronic inhibition of HDAC activity.

HDAC Inhibition Intercepts and Erases the Epileptic State. The ultimate goal of epilepsy therapies is curing epilepsy, with complete erasure of the epileptogenic state from the brain, such that additional treatments are not needed. Thus far, this goal has not been met with success since there has been no drug that can truly prevent epilepsy. To our knowledge, no compounds have been identified that can erase or promote unwiring of the epileptogenic state. Unexpectedly, our studies uncovered that butyrate has the potential to reverse or erase the hyperexcitability state of epileptic animals. In fully kindled epileptic animals, butyrate treatment and discontinuation caused seizure severity to actually decrease rather than remain at an epileptic steady state. This dramatic outcome indicates that butyrate treatment modified the persistence of the epileptogenic state, a phenomenon called reversal or erasure of the disease. The potential mechanisms underlying such erasure or regression of epilepsy remain elusive. Epigenetic HDAC inhibition by butyrate could potentially limit the proliferation of genes involved in structural aspects of neuronal circuitry involved in seizure generation or propagation; this could reduce the severity of future epileptogenic response or facilitate unwiring of hyperexcitable neuronal networks in epilepsy. Further studies are warranted to advance this novel phenomenon with other HDAC inhibitors.

HDAC Inhibition with Concurrent Antiseizure Drug Promotes Further Modification of Epileptogenesis. Neurosteroids are powerful allosteric modulators of tonic inhibition with strong antiseizure properties (Reddy, 2010; Reddy and Estes, 2016). A combination of both epigenetic and neurosteroid treatments could provide the best of both worlds with strong acute action and effective long-term antiepileptogenic management. Neurosteroids have potent seizure suppression when administered shortly before stimulation, while an epigenetic agent such as sodium butyrate offers long-term inhibition of the kindling process. In the present study, coadministration of butyrate and GX drastically slowed the progress of epileptogenesis when injected daily alongside stimulations. This result persisted even after withdrawal of both drugs, with the combination treatment displaying a lasting seizure protection more potent than that of butyrate or GX alone. A combination therapy of butyrate and GX facilitated effective disease modification as reflected by nearly complete prevention of hippocampus mossy fiber sprouting, which is widely-considered a hallmark morphologic index of epileptogenesis.

Therapeutic Implications of HDAC Inhibition for Curing Epilepsy. Currently, there is no known treatment that can prevent epilepsy from developing in individuals at risk. A variety of HDAC inhibitors are currently being used in clinical trials for cancer and neurologic diseases. They hold promising potential as effective epileptogenic inhibitors. This targeted strategy has many advantages, including a wide availability of clinically approved HDAC 
A
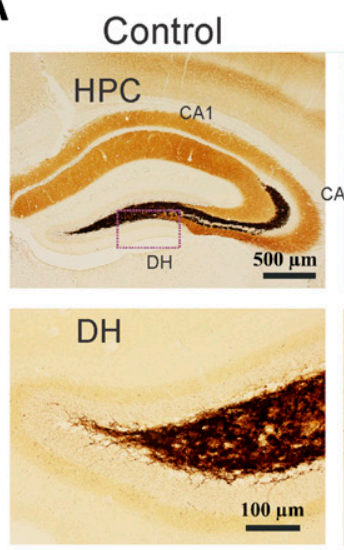
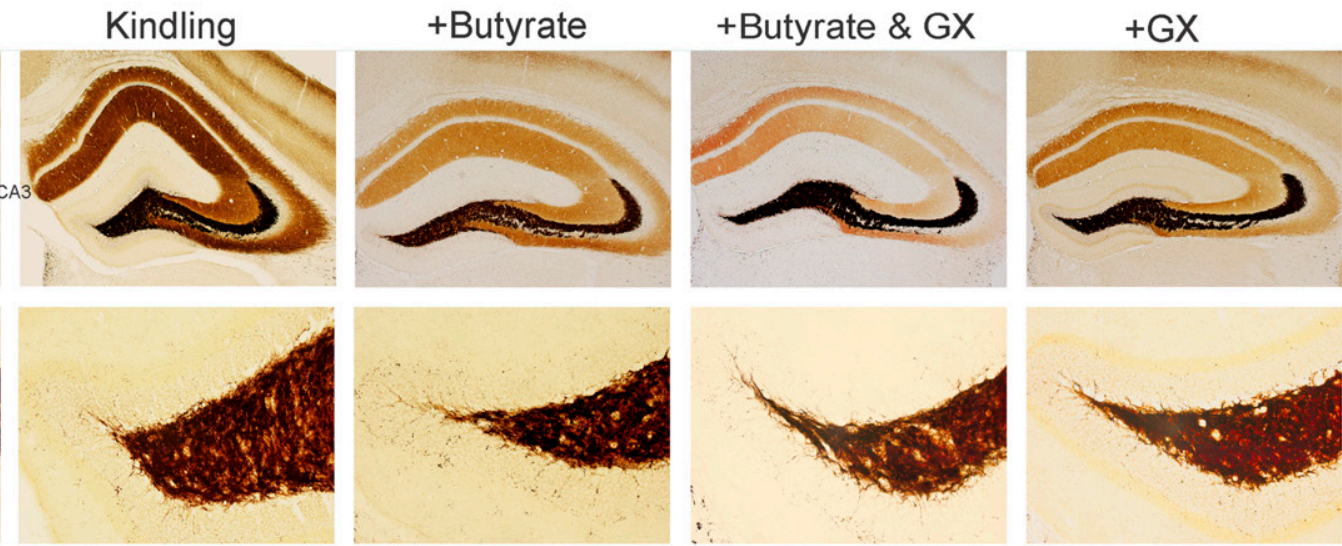

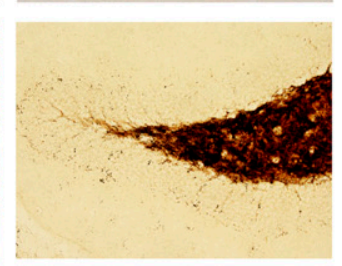

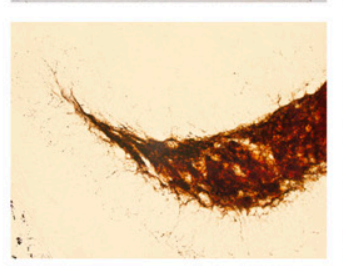

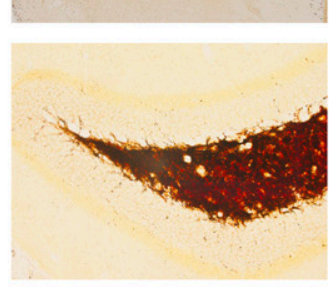

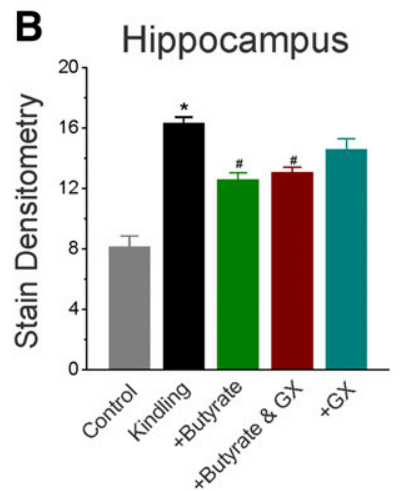
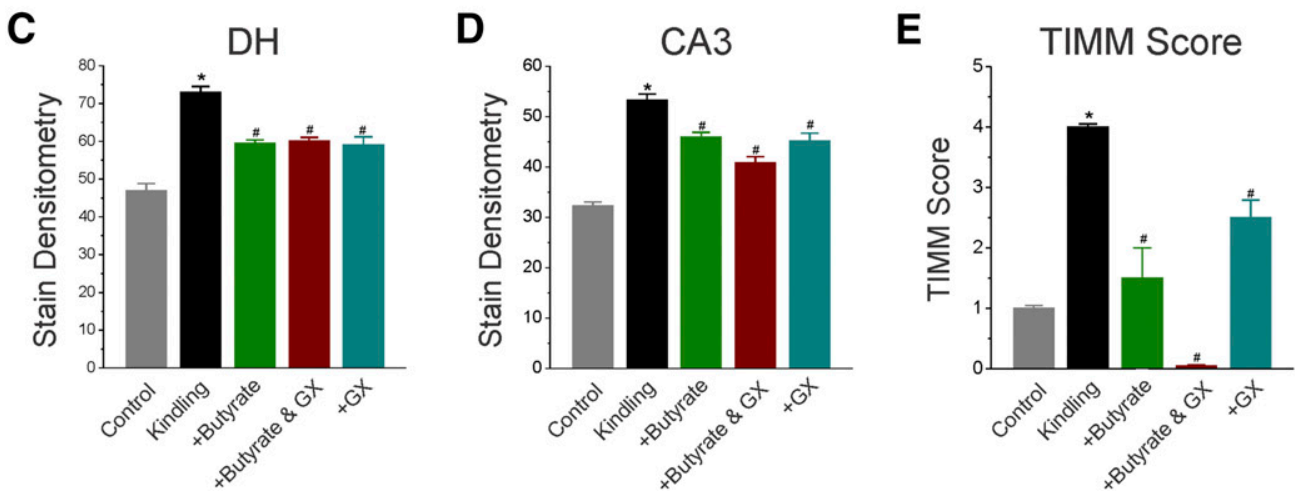

Fig. 10. HDAC inhibition reduces epileptogenic mossy fiber sprouting in the hippocampus in epileptic mice. At the completion of chronic treatment experiments, animals from each kindling group were perfused and brain slices from the hippocampus were prepared. Slices were then Timm stained and fiber sprouting was quantified through densitometric analysis. (A) The top row of images is an expanded view of the entire hippocampus, while the bottom row is a $5 \times$ zoom onto the dentate hilus (DH) subfield. (B) Butyrate treatment significantly limited fiber sprouting in the hippocampus during kindling. (C) Butyrate treatments greatly reduced fiber sprouting in the DH subfield. (D) Butyrate and GX treatments reduced fiber sprouting in the CA3, but did not prevent the bulk of sprouting. (E) Butyrate and/or GX treatment during kindling significantly reduced Timm scores in the hippocampus, but butyrate-treated groups showed greater reduction than the GX group. ${ }^{*} P<0.05$ vs. naive (nonkindled) control group; \#P<0.05 vs. kindling (untreated) group.

inhibitors, a concrete strategy based on the pathogenic mechanism involved in epileptogenesis, and the potential for preventative, long-term protection rather than symptomatic treatment. Epigenetic HDAC intervention appears to be a rational strategy for preventing epilepsy because it targets the primary pathway that initially triggers the numerous downstream neuronal events mediating epileptogenesis. The mechanisms underlying epigenetic regulation of epileptogenesis remain unclear, but appear to be complex and multifaceted (Walker et al., 2002; Reddy et al., 2010; Reddy and Mohan, 2011). Neuronal injury activates diverse signaling events, such as inflammation, apoptosis, neurogenesis, and synaptic plasticity, which eventually lead to structural and functional changes in neuronal circuits (Löscher, 2002; Pitkänen et al., 2015). These changes eventually manifest as abnormal network hyperexcitability and spontaneous seizures. HDACs, as upstream regulators of transcription of genes, can dynamically regulate a variety of downstream signaling pathways involved in epileptogenesis, including gene networks that govern the circuit excitability and synaptic function (Crosio et al., 2003; Dong et al., 2007; Jessberger et al., 2007; Younus and Reddy, 2017b). The target mRNA and protein abundance assays are being performed to uncover the antiepileptogenic mechanism underlying the HDAC inhibition. Specific HDAC components may serve as valid biomarkers for epileptogenesis monitoring. One of the mechanisms of the ketogenic diet, which also has antiepileptogenic activity, is thought to be based on HDAC inhibition via $\beta$-hydroxybutyrate (Moreno and Mobbs, 2017; Wang et al., 2017). These findings are consistent with our findings on HDAC interruption of the antiepileptogenic process. Thus, the HDAC inhibitors may hold great potential as antiepileptogenic agents for curing epilepsy. Other epigenetic mechanisms, including DNA methylation, microRNA-based transcriptional control, and bromodomain activity, may alter epileptogenesis by exerting their regulatory effects on neuronal plasticity (Younus and Reddy, 2017b). Although such targets represent alternatives for antiepileptogenic interventions, there are currently few approved drugs with acceptable safety profiles for experimental investigations.

In conclusion, the powerful inhibition of kindling epileptogenesis progression under both subchronic and chronic HDAC inhibition by sodium butyrate protocols suggests a critical role of the epigenetic HDAC pathway in the development and persistence of epilepsy. This was associated with reduced neuronal fiber sprouting in the hippocampus. HDAC inhibition caused long-lasting disease modification and strikingly reversed the kindling epileptogenic state, 
which is a unique aspect of epigenetic targeting compared with traditional antiseizure treatments. These findings, therefore, envisage a novel therapy for preventing or curing epilepsy by targeting specific epigenetic HDAC pathways in the brain.

\section{Acknowledgments}

We thank Daniel Jones and Amir Bhimani for outstanding kindling assistance and Xin Wu and Victoria Golub for help with the HDAC assays.

\section{Authorship Contributions}

Participated in research design: S.D. Reddy, D.S. Reddy.

Conducted experiments: S.D. Reddy, Clossen, D.S. Reddy.

Performed data analysis: S.D. Reddy, D.S. Reddy.

Wrote or contributed to the writing of the manuscript: S.D. Reddy, D.S. Reddy.

\section{References}

Albertson TE, Joy RM, and Stark LG (1984) A pharmacological study in the kindling model of epilepsy. Neuropharmacology 23:1117-1123.

Borthakur A, Saksena S, Gill RK, Alrefai WA, Ramaswamy K, and Dudeja PK (2008) Regulation of monocarboxylate transporter 1 (MCT1) promoter by butyrate in human intestinal epithelial cells: involvement of NF- $\kappa \mathrm{B}$ pathway. $J$ Cell Biochem 103:1452-1463.

Brandt C, Glien M, Gastens AM, Fedrowitz M, Bethmann K, Volk HA, Potschka H, and Löscher W (2007) Prophylactic treatment with levetiracetam after status epilepticus: lack of effect on epileptogenesis, neuronal damage, and behavioral alterations in rats. Neuropharmacology 53:207-221.

Carey N and La Thangue NB (2006) Histone deacetylase inhibitors: gathering pace. Curr Opin Pharmacol 6:369-375.

Cavazos JE, Golarai G, and Sutula TP (1991) Mossy fiber synaptic reorganization induced by kindling: time course of development, progression, and permanence. $J$ Neurosci 11:2795-2803.

Clossen BL and Reddy DS (2017) Novel therapeutic approaches for diseasemodification of epileptogenesis for curing epilepsy. Biochim Biophys Acta 1863: $1519-1538$.

Crosio C, Heitz E, Allis CD, Borrelli E, and Sassone-Corsi P (2003) Chromatin remodeling and neuronal response: multiple signaling pathways induce specific histone H3 modifications and early gene expression in hippocampal neurons. J Cell Sci 116:4905-4914.

Davie JR (2003) Inhibition of histone deacetylase activity by butyrate. J Nutr 133 (Suppl 7):2485S-2493S

Deutsch SI, Rosse RB, Long KD, Gaskins BL, Burket JA, and Mastropaolo J (2008) Sodium butyrate, an epigenetic interventional strategy, attenuates a stressinduced alteration of MK-801's pharmacologic action. Eur Neuropsychopharmacol 18:565-568.

Dong E, Guidotti A, Grayson DR, and Costa E (2007) Histone hyperacetylation induces demethylation of reelin and $67-\mathrm{kDa}$ glutamic acid decarboxylase promoters. Proc Natl Acad Sci USA 104:4676-4681.

Ferrante RJ, Kubilus JK, Lee J, Ryu H, Beesen A, Zucker B, Smith K, Kowall NW, Ratan RR, Luthi-Carter R, et al. (2003) Histone deacetylase inhibition by sodium butyrate chemotherapy ameliorates the neurodegenerative phenotype in Huntington's disease mice. J Neurosci 23:9418-9427.

Franklin KBJ and Paxinos G (1997) The Mouse Brain in Stereotaxic Coordinates, 1st ed, Academic Press, New York.

Garcia-Bassets I, Kwon YS, Telese F, Prefontaine GG, Hutt KR, Cheng CS, Ju BG, Ohgi KA, Wang J, Escoubet-Lozach L, et al. (2007) Histone methylation-dependent mechanisms impose ligand dependency for gene activation by nuclear receptors. Cell 128:505-518.

Goddard GV, McIntyre DC, and Leech CK (1969) A permanent change in brain function resulting from daily electrical stimulation. Exp Neurol 25:295-330.

Gorter JA, van Vliet EA, and Lopes da Silva FH (2016) Which insights have we gained from the kindling and post-status epilepticus models? J Neurosci Methods 260:96-108.

Gray SG and Ekström TJ (2001) The human histone deacetylase family. Exp Cell Res 262:75-83.

Gurvich N, Tsygankova OM, Meinkoth JL, and Klein PS (2004) Histone deacetylase is a target of valproic acid-mediated cellular differentiation. Cancer Res 64: 1079-1086.

He XP, Kotloski R, Nef S, Luikart BW, Parada LF, and McNamara JO (2004) Conditional deletion of TrkB but not BDNF prevents epileptogenesis in the kindling model. Neuron 43:31-42.

Heng K, Haney MM, and Buckmaster PS (2013) High-dose rapamycin blocks mossy fiber sprouting but not seizures in a mouse model of temporal lobe epilepsy. Epilepsia 54:1535-1541.

Hesdorffer DC, Beck V, Begley CE, Bishop ML, Cushner-Weinstein S, Holmes GL, Shafer PO, Sirven JI, and Austin JK (2013) Research implications of the Institute of Medicine Report, epilepsy across the spectrum: promoting health and understanding. Epilepsia 54:207-216.

Jacobs MP, Leblanc GG, Brooks-Kayal A, Jensen FE, Lowenstein DH, Noebels JL, Spencer DD, and Swann JW (2009) Curing epilepsy: progress and future directions. Epilepsy Behav 14:438-445.
Jessberger S, Nakashima K, Clemenson GD, Jr, Mejia E, Mathews E, Ure K, Ogawa S, Sinton CM, Gage FH, and Hsieh J (2007) Epigenetic modulation of seizure-induced neurogenesis and cognitive decline. $J$ Neurosci 27 $5967-5975$.

Kim SW, Hooker JM, Otto N, Win K, Muench L, Shea C, Carter P, King P, Reid AE, Volkow ND, et al. (2013) Whole-body pharmacokinetics of HDAC inhibitor drugs, butyric acid, valproic acid and 4-phenylbutyric acid measured with carbon-11 labeled analogs by PET. Nucl Med Biol 40:912-918

Kobow K and Blümcke I (2011) The methylation hypothesis: do epigenetic chromatin modifications play a role in epileptogenesis? Epilepsia $\mathbf{5 2}$ (Suppl 4) $15-19$

Kouzarides T (2007) Chromatin modifications and their function. Cell 128:693-705 Löscher W (2002) Animal models of epilepsy for the development of antiepileptogenic and disease-modifying drugs. A comparison of the pharmacology of kindling and post-status epilepticus models of temporal lobe epilepsy. Epilepsy Res 50:105-123.

Löscher W, Hönack D, and Rundfeldt C (1998) Antiepileptogenic effects of the novel anticonvulsant levetiracetam (ucb L059) in the kindling model of temporal lobe epilepsy. J Pharmacol Exp Ther 284:474-479.

Lothman EW and Williamson JM (1993) Rapid kindling with recurrent hippocampal seizures. Epilepsy Res 14:209-220.

Moreno CL and Mobbs CV (2017) Epigenetic mechanisms underlying lifespan and age-related effects of dietary restriction and the ketogenic diet. Mol Cell Endocrinol 455:33-40

McNamara JO, Morrisett R, and Nadler JV (1992) Recent advances in understanding mechanisms of the kindling model. Adv Neurol 57:555-560.

Minamiyama M, Katsuno M, Adachi H, Waza M, Sang C, Kobayashi Y, Tanaka F, Doyu M, Inukai A, and Sobue G (2004) Sodium butyrate ameliorates phenotypic expression in a transgenic mouse model of spinal and bulbar muscular atrophy. Hum Mol Genet 13:1183-1192.

Morris MJ, Karra AS, and Monteggia LM (2010) Histone deacetylases govern cellular mechanisms underlying behavioral and synaptic plasticity in the developing and adult brain. Behav Pharmacol 21:409-419.

Nian H, Bisson WH, Dashwood WM, Pinto JT, and Dashwood RH (2009) $\alpha$-Keto acid metabolites of organoselenium compounds inhibit histone deacetylase activity in human colon cancer cells. Carcinogenesis 30:1416-1423.

Pajak B, Orzechowski A, and Gajkowska B (2007) Molecular basis of sodium butyrate-dependent proapoptotic activity in cancer cells. Adv Med Sci 52:83-88.

Pitkänen A and Lukasiuk K (2011) Mechanisms of epileptogenesis and potential treatment targets. Lancet Neurol 10:173-186.

Pitkänen A, Nehlig A, Brooks-Kayal AR, Dudek FE, Friedman D, Galanopoulou AS, Jensen FE, Kaminski RM, Kapur J, Klitgaard H, et al. (2013) Issues related to development of antiepileptogenic therapies. Epilepsia 54 (Suppl 4):35-43.

Pitkänen A, Lukasiuk K, Dudek FE, and Staley KJ (2015) Epileptogenesis. Cold Spring Harb Perspect Med 5 (a022822):1-15.

Qureshi IA and Mehler MF (2010a) Epigenetic mechanisms underlying human epileptic disorders and the process of epileptogenesis. Neurobiol Dis 39:53-60.

Qureshi IA and Mehler MF (2010b) Genetic and epigenetic underpinnings of sex differences in the brain and in neurological and psychiatric disease susceptibility. Prog Brain Res 186:77-95.

Racine RJ (1972) Modification of seizure activity by electrical stimulation. II. Motor seizure. Electroencephalogr Clin Neurophysiol 32:281-294.

Rajendran P, Delage B, Dashwood WM, Yu TW, Wuth B, Williams DE, Ho E, and Dashwood RH (2011) Histone deacetylase turnover and recovery in sulforaphane-treated colon cancer cells: competing actions of 14-3-3 and Pin1 in HDAC3/SMRT corepressor complex dissociation/reassembly. Mol Cancer 10:68.

Rao MS, Hattiangady B, Reddy DS, and Shetty AK (2006) Hippocampal neurodegeneration, spontaneous seizures, and mossy fiber sprouting in the F344 rat model of temporal lobe epilepsy. J Neurosci Res 83:1088-1105.

Ratzliff Ad, Santhakumar V, Howard A, and Soltesz I (2002) Mossy cells in epilepsy: rigor mortis or vigor mortis? Trends Neurosci 25:140-144.

Ravizza T, Balosso S, and Vezzani A (2011) Inflammation and prevention of epileptogenesis. Neurosci Lett 497:223-230.

Reddy DS (2010) Neurosteroids: endogenous role in the human brain and therapeutic potentials. Prog Brain Res 186:113-137.

Reddy DS and Estes WA (2016) Clinical potential of neurosteroids for CNS disorders. Trends Pharmacol Sci 37:543-561.

Reddy DS, Gould J, and Gangisetty O (2012) A mouse kindling model of perimenstrual catamenial epilepsy. J Pharmacol Exp Ther 341:784-793.

Reddy DS, Gangisetty O, and Briyal S (2010) Disease-modifying activity of progesterone in the hippocampus kindling model of epileptogenesis. Neuropharmacology 59:573-581.

Reddy DS and Jian K (2010) The testosterone-derived neurosteroid androstanediol is a positive allosteric modulator of $\mathrm{GABA}_{\mathrm{A}}$ receptors. J Pharmacol Exp Ther 334: 1031-1041.

Reddy DS and Mohan A (2011) Development and persistence of limbic epileptogenesis are impaired in mice lacking progesterone receptors. $J$ Neurosci 31:650-658.

Reddy DS and Rogawski MA (2010) Ganaxolone suppression of behavioral and electrographic seizures in the mouse amygdala kindling model. Epilepsy Res 89:254-260.

Sankar R, Auvin S, Kwon YS, Pineda E, Shin D, and Mazarati A (2010) Evaluation of development-specific targets for antiepileptogenic therapy using rapid kindling. Epilepsia 51 (Suppl 3):39-42.

Silver JM, Shin C, and McNamara JO (1991) Antiepileptogenic effects of conventional anticonvulsants in the kindling model of epilespy. Ann Neurol 29:356-363.

Takuma K, Hara Y, Kataoka S, Kawanai T, Maeda Y, Watanabe R, Takano E, Hayata-Takano A, Hashimoto H, Ago Y, et al. (2014) Chronic treatment with valproic acid or sodium butyrate attenuates novel object recognition deficits and hippocampal dendritic spine loss in a mouse model of autism. Pharmacol Biochem Behav 126:43-49.

Verdone L, Caserta M, and Di Mauro E (2005) Role of histone acetylation in the control of gene expression. Biochem Cell Biol 83:344-353. 
Ververis K, Hiong A, Karagiannis TC, and Licciardi PV (2013) Histone deacetylase inhibitors (HDACIs): multitargeted anticancer agents. Biologics 7:47-60.

Walker MC, White HS, and Sander JW (2002) Disease modification in partial epilepsy. Brain 125:1937-1950.

Wang X, Wu X, Liu Q, Kong G, Zhou J, Jiang J, Wu X, Huang Z, Su W, and Zhu Q (2017) Ketogenic metabolism inhibits histone deacetylase (HDAC) and reduces oxidative stress after spinal cord injury in rat. Neuroscience [published ahead of print].

Younus I and Reddy DS (2017a) A resurging boom in new drugs for epilepsy and brain disorders. Expert Rev Clin Pharmacol 23:1-19.
Younus I and Reddy DS (2017b) Epigenetic interventions for epileptogenesis: a new frontier for curing epilepsy. Pharmacol Ther 177:108-122.

Address correspondence to: Dr. Doodipala Samba Reddy, Department of Neuroscience and Experimental Therapeutics, College of Medicine, Texas A\&M University Health Science Center, 2008 Medical Research and Education Building, 8447 Riverside Parkway, Bryan, TX 77807-3260. E-mail: reddy@ medicine.tamhsc.edu 